\title{
International
}

\section{Journal of Medical and Exercise Science}

(Multidisciplinary, Peer Reviewed and Indexed Journal)

\section{ORIGINAL ARTICLE}

\section{EFFECTIVENESS OF MOBILIZATION WITH MOVEMENT AND MYOFASCIAL RELEASE TECHNIQUE IN IMPROVING PAIN FREE GRIP STRENGTH, PRESSURE PAIN THRESHOLD AND FUNCTIONAL DISABILITY IN SUBJECTS WITH RECURRENT CHRONIC LATERAL EPICONDYLALGIA}

Search engine:

www.ijmaes.org

\section{ANUSHA M S ${ }^{1}$, ANDREWS MILTON ${ }^{2}$}

\section{Corresponding Author:}

${ }^{1}$ MPT Musculoskeletal \& Sports - student , Bethany Navajeevan College of Physiotherapy, Thiruvananthapuram, Kerala, India. Mail id: anushams48@gmail.com

Co Author:

${ }^{2}$ Professor, Bethany Navajeevan College of Physiotherapy, Thiruvananthapuram, Kerala, India. ABSTRACT

Background: Recurrent musculoskeletal pain has an apparent repercussion on health care costs, employment productivity and quality of life. Lateral epicondylalgia, is a common recurrent musculoskeletal complaint that is often confronted by physical therapists. Pain, reduced grip strength and functional disability are main problems seen in chronic lateral epicondylalgia patients (CLE). The complexity in pathophysiology of CLE is reflected by the lack of consensus on management and remains a therapeutic challenge. Purpose of the study was to investigate the effectiveness of Mulligan's mobilization with movement and myofascial release technique on pain, pain free grip strength and functional disability in recurrent chronic lateral epicondylalgia patients. Methods: Thirty subjects within age group 25-50 fulfilled the inclusion criteria were recruited in this study. The subjects were divided into two groups; Group A (Experimental) and Group B (Control). Group A underwent Mulligan's Mobilization with movement, myofascial release technique and Conventional treatment, Group $B$ underwent Conventional treatment only. Interventions were conducted over a period of 4 weeks, 3 sessions per week, 1 session per day. All subjects were assessed for pain free grip strength using hand dynamometer, functional disability using Patient rated tennis elbow evaluation questionnaire and pressure pain threshold using pressure algometer before and after intervention. Results: Mulligan's MWM combined with myfascial release technique and conservative treatment is more effective in treating recurrent chronic lateral epicondylalgia. Conclusion: This study asserts a multimodal treatment approach for the management of recurrent chronic lateral epicondylalgia by relieving myofascial, articular and nervous system impairments.

Keywords: Chronic lateral epicondylalgia; Mulligan's mobilization with movement; Myofascial release technique; Pressure pain threshold; Pain free grip strength.

Received on $09^{\text {th }}$ August 2017, Revised $19^{\text {th }}$ August 2017, Accepted on $28^{\text {th }}$ August 2017 


\section{INTRODUCTION}

Lateral epicondylalgia is the most common affliction of the elbow which results in considerable morbidity and financial cost in the healthcare industry ${ }^{1}$. This prevalent painful disabling musculoskeletal disorder is characterized by pain in the external aspect of elbow exacerbated during elbow extension with the wrist in flexed position or by resisted extension movement of the wrist with the elbow in extension and during grasping and gripping activities. This injury is a major challenge, as it is difficult to treat, prone to recurrence and may last for several weeks or months, with an average duration of a typical episode which has been reported to be between six months to two years ${ }^{2}$.

Morris and Major described it as 'lawn tennis elbow'. Runge described it as 'writer's cramp'. Lateral epicondylitis is an inappropriate term as the primary pathology is due to collagen disorganisation rather than inflammatory changes $^{3}$. The preferred nomenclature is lateral epicondylalgia, as the suffix '-algia' denotes pain; the pathophysiology of the condition is less commonly inflammation or degeneration than it is predominantly hyperalgesia and pain ${ }^{4}$. If patient have symptoms more than 3 months, LE can be referred as chronic.

Epidemiological studies indicates LE typically affects people between the ages of 35 and 60 with incidence of 4-7 per 1000 per year in the general population ${ }^{5}$ with the annual incidence of $1-3 \%$ in the general population which increases to $19 \%$ in $30-60$ years old population and up to $9.1 \%$ of tennis players. Shiri et al reported on a population study that the prevalence of LE is $1.3 \%{ }^{6}$.
The prevalence was equal in men and women and was highest in those aged $45-54$ years old and appears to be more long standing and severe in women ${ }^{6}$. Changes of racket, altering stroke technique, and exercising to build-up muscle strength have been reported successful in alleviating the symptoms of LE and preventing recurrence ${ }^{7}$. It is found that physical load factors, smoking and obesity are strong determinants of epicondylosis ${ }^{6}$.

Major complaints of LE patients are pain, functional difficulty and reduced grip strength affecting activities of daily living related to wrist and forearm movements. The grip strength is affected in chronic cases mainly due to 2 factors, voluntary deterioration of effort of patients to avoid pain and due to wasting of forearm muscles as seen in long standing conditions ${ }^{8}$.

LE is thought to result from overuse of the ECRB muscle by repetitive microtrauma resulting in a primary tendinosis of the ECRB, with or without involvement of the EDC. Results from cytological studies shows that is a non-inflammatory and degenerative process leading to a disarray and untimely tendon repair termed 'angiofibroblastic tendinosis'12 which was characterised by key changes like increased cell numbers, increased production of ground substance, neovascularization, increased neurochemical concentration, disorganised and immature collagen. All these changed occurs if the tendon fails to heal properly after injury or due to overuse. Studies found that it is difficult to completely exclude an inflammatory component in LE due to a number of factors such as clinical observation of swelling and warmth, the proven positive effect of anti-inflammatory drugs and corticosteroids. It still remains unknown 
whether inflammation precedes degeneration in the more acute phase of this condition. Pain mechanisms in CLE are due to central sensitization and the triggering of nociceptors by neurotransmitters or biochemical irritation due to the noxious products of cellular activity. Substance $P$ found in the origin of ECRB tendon, has powerful influence on pain modulation in central nervous system.

LE is clinically diagnosed by mill's test or cozen's test. The natural course of the condition seems to be favourable, with spontaneous recovery within 1-2 years in 80$90 \%$ of the patients; however there is very little scientific data available on the natural history of the disease. Although often described as self-limiting, overuse chronic type injuries are not always self-limiting and can result in prolonged symptoms and be prone to recurrences.

There have been over 40 treatments used for lateral epiondylalgia, but currently, no general consensus exists as the most appropriate management for Lateral Epicondylalgia. Very few studies are available for the myofascial release technique and no studies are available for the combined treatment of Mulligan's mobilization with movement and myofascial release technique in recurrent chronic lateral epiondylalgia. Hence, a need arises to rule out the usefulness of, the addition of myofascial release technique with Mulligan's MWM and conventional treatment for patients with recurrent chronic lateral epicondylalgia. This study investigates the effectiveness of MWM and myofascial release technique on pain, grip strength and functional disability in recurrent chronic lateral epicondylalgia patients.
Mulligan's Mobilization with Movement (MWM) is a technique developed by Mulligan for treating CLE. MWM is a form of manual therapy that includes a sustained lateral glide to the elbow joint with concurrent physiological movement ${ }^{9}$. This mobilization technique is often used to correct the faulty position of the elbow joint is being widely used in management of musculoskeletal disorders. Miller ${ }^{5}$ described in his case report the use of the MWM for lateral epicondylalgia resulting in reduced pain, improvement of painfree grip strength (PFGS), and increased ability to tolerate resisted isometric wrist extension.

Myofascial release technique is the application of a low load, long duration stretch to the myofascial complex in order to relieve fascial constrictions ${ }^{10,11}$. Muscle imbalance is one of the important aetiological factors in chronic lateral epicondylalgia. So application of MFR intended to restore optimal length, decrease pain and improve function in CLE.

\section{Aim of the study:}

The aim of this study is to find out the effectiveness of mulligan's mobilization with movement and myofascial release technique in improving pain free grip strength, pressure pain threshold and functional disability in recurrent chronic lateral epicondylalgia patients.

\section{Objectives of the study :}

1)To determine the effectiveness of mobilization with movement technique and myofascial release technique in improving pain free grip strength using hand dynamometer in subjects with recurrent chronic lateral epicondylalgia.

2)To determine the effectiveness of mobilization with movement technique and myofascial release technique in improving 
pressure pain threshold using pressure algometer in subjects with recurrent chronic lateral epicondylalgia.

3)To determine the effectiveness of mobilization with movement technique and myofascial release technique in improving functional disability using Patient Rated Tennis Elbow Evaluation Questionnaire in subjects with recurrent chronic lateral epicondylalgia.

Null hypothesis: There will be no significant difference in pain free grip strength, pressure pain threshold and functional disability after the application of mobilization with movement technique and myofascial release technique in subjects with recurrent chronic lateral epicondylalgia.

Alternative hypothesis: There will be significant difference in pain free grip strength, pressure pain threshold and functional disability after the application of mobilization with movement technique and myofascial release technique in subjects with recurrent chronic lateral epicondylalgia.

\section{METHODOLOGY}

Study Design: Pre versus posttest experimental study design.

Study Setting: Study conducted at Physiotherapy department of Bethany Navajeevan College of Physiotherapy.

Sample Size: Total 30 samples were selected for this study, and then equally divided in to 15 subjects for Group A and Group B.

Inclusion Criteria: Age 25-50 years, both males and females, patients with a diagnosis of lateral epicondylalgia based on Southampton examination criteria, Lateral Epicondylalgia greater than 3 months with more than single episode, unilateral involvement, Numerical Pain Rating Score 4 to 8 , tenderness over the lateral elbow region.

Exclusion Criteria: Bilateral lateral epicondylalgia, surgery for lateral epicondylitis within the last twelve months, recent fracture of either radius, ulna and humerus, rheumatoid disease, elbow instability, neurologic impairments, cervical radiculopathy, cardiovascular diseases, corticosteroid injection within 6 months, osteoporosis, ossification and calcification of soft tissue, malignancies.

Sampling: Purposive Sampling method used to select the samples in this study.

Study Duration: The study was conducted over a period of 9 months

\section{Outcome Measures:}

a.Pain free grip strength evaluated using Hand Dynamometer

b.Pressure pain threshold evaluated using Pressure Algometer

c.Functional disability evaluated using Patient Rated Tennis Elbow Evaluation Questionnaire.

\section{Statistical Tools:}

1. Wilcoxon Signed Rank Test

2. Mann Whitney U Test

Procedure: 30 subjects between the age group of 25-50 years who fulfilled the inclusion criteria were included in the study. The duration of LE was more than 3 months and recurrent episode. A standardized assessment conducted prior to their inclusion in the study. Subjective assessment of the patient was done which includes name, age, gender, chief 
complaints, duration of the condition and history of the patient. The subjects were divided into two groups, i.e., Group A (experimental group) and Group B (control group), with 15 subjects each.

This study was comprised of 3 steps,

1. Pre-testing

2. Training Intervention

\section{Post-testing}

Once the subjects were classified into these groups, an informed consent collected from them. Pretest was conducted on Group A and Group B using Hand Dynamometer for measuring Pain Free Grip Strength, Pressure Algometer for measuring pressure pain threshold and Patient Rated Tennis Elbow Evaluation Questionnaire for measuring functional disabiltiy.

After a brief demonstration about mulligan's mobilization with movement technique, myofascial release technique and conventional physiotherapy, Group A subjects were subjected to mobilization with movement technique, myofascial release technique and conventional physiotherapy including pulsed ultrasound therapy, stretching and strengthening of wrist flexors and extensors for 3 days per week for a period of 4 weeks with one session per day.

After a brief demonstration about conventional physiotherapy, Group B subjects were subjected to conventional physiotherapy including pulsed ultrasound therapy, stretching and strengthening of wrist flexors and extensors for 3 days per week for a period of 4 weeks with one session per day.
Posttest was conducted on Group A and Group $B$ by Hand Dynamometer for measuring Pain Free Grip Strength, Pressure Algometer for measuring pressure pain threshold and Patient Rated Tennis Elbow Questionnaire for measuring functional disability after the treatment program.

\section{TRAINING INTERVENTION}

\section{MULLIGAN MOBILIZATION WITH MOVEMENT TECHNIQUE ${ }^{22}$}

Mulligans mobilization with movement, which involves the application of lateral glide to the elbow joint, was sustained while an exercise, activity or movement was performed. Mobilization belt was placed around the patient's proximal forearm and across the therapist's shoulder while the distal humerus was stabilized with one hand. Lateral glide was applied to the forearm through belt and sustained for about 5-10 sec. While the patient performed repeated wrist extension. The lateral glide was released after the subject had achieved the pain free wrist extension. Six repetitions were performed with a $15 \mathrm{sec}$. rest interval between repetitions.

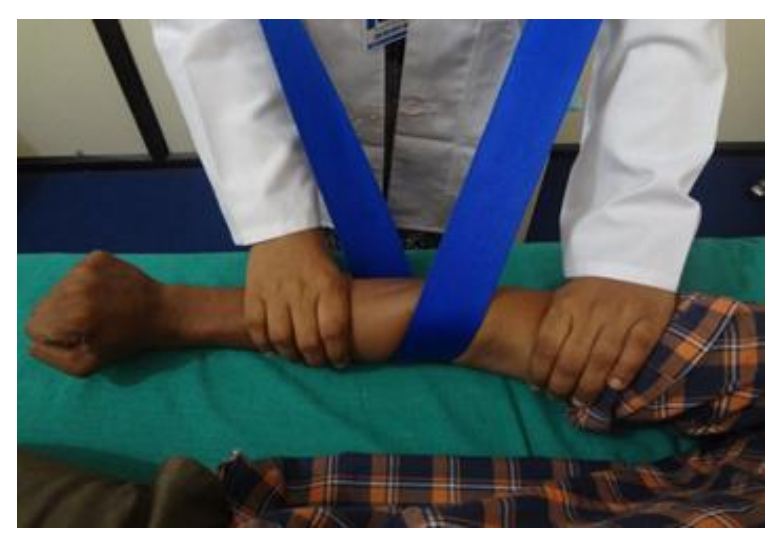

Figure 1: Subject receiving Mulligan's Mobilization with movement 


\section{MYOFASCIAL RELEASE TECHNIQUE ${ }^{11}$}

The subjects were in supine with affected side shoulder rotate internally, elbow flexion to around $15^{\circ}$ and pronation, palm resting flat on table. Therapist stands at the side of table near shoulder and facing ipsilateral hand.

Procedure 1: Treating from the common extensor tendon to the extensor retinaculum of the wrist, the therapist began on the humerus, just proximal to the lateral epicondyle. The therapist used the fingertips to engage the periosteum and carried this contact inferior to the common extensor tendon and then down to the extensor retinaculum of the wrist. Patients were trained to slowly flex and extend the elbow within an easy range of $5^{\circ}$ to $10^{\circ}$ during this procedure ( $5 \mathrm{~min}, 2$ repetitions).

Procedure 2: Treating through the periosteum of the ulna, the therapist used the knuckles of the hand to work over the periosteum of the ulna. Patients were trained to do alternating ulnar and radial deviation of the wrist while periosteum of ulna being engaged. (5min, 2 repetitions).

Procedure 3: Spreading the radius from the ulna, the therapist contacted the head of the ulna with the finger pads of one hand and the dorsal tubercle of radius with the pads of the other. The therapist was engaged through to the periosteum and put a line of tension in a lateral and distal direction. This was carried for just a few centimeters with a firm intent to spread the bones apart ( $5 \mathrm{~min}, 2$ repetitions). Total 30 minutes session, 3 times a week for 4 weeks.

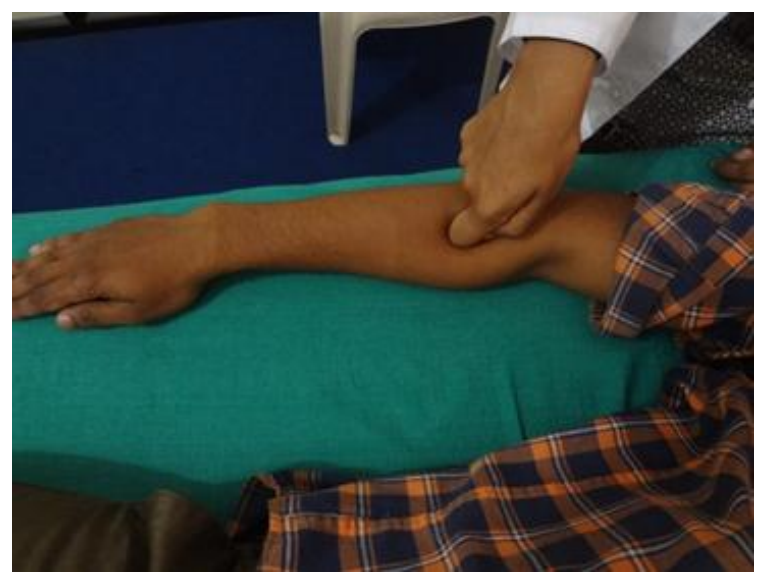

Figure 2: Subject receiving Myofascial release technique

\section{ULTRASOUND THERAPY21}

Following MWM ,Group A subjects were subjected to the pulsed ultrasound therapy at tenoperiosteal junction of the extensor carpi radialis brevis with $1: 4$ Pulse Ratio of $1 \mathrm{MHz}$ at $1.5 \mathrm{~W} / \mathrm{cm} 2$ for 5 minutes. Total: 3 Session per Week, Total of 12 sessions.

\section{STRETCHING EXERCISES ${ }^{21}$}

Stretching Exercises were given with patient seated and forearm pronated, elbow extended; the wrist being palmar- flexed using the other hand of patient or with the help of wall. This was held for 30 seconds and then released. Total: 10 stretch per session, Daily One session

\section{STRENGTHENING EXERCISES ${ }^{21}$}

Strengthening Exercises were given with patient in seating position and isometric contractions with the elbow flexed to $90^{\circ}$, with the hand of unaffected arm applying manual resistance over the dorsum of the supinated arm of affected side. Pain free isometric contraction of the wrist extensors was initiated and held for 5 to 10 seconds. Total: 15 contractions per session, Daily One Session. 


\section{RESULTS}

GROUP A (EXPERIMENTAL GROUP)

\begin{tabular}{|c|c|c|c|c|c|c|}
\hline \multirow{2}{*}{$\begin{array}{l}\text { OUT COME } \\
\text { MEASURES }\end{array}$} & \multicolumn{2}{|c|}{ MEAN } & \multicolumn{2}{|c|}{ STANDARD DEVIATION } & \multirow{2}{*}{ Z VALUE } & \multirow{2}{*}{ P VALUE } \\
\hline & PRE TEST & POST TEST & PRE TEST & POST TEST & & \\
\hline PRTEE & 42.63 & 31.03 & 13.102 & 12.709 & -3.415 & .000 \\
\hline PFGS & 12.80 & 20.47 & 3.688 & 3.907 & -3.423 & .000 \\
\hline PPT & 2.20 & 3.09 & .469 & .425 & -3.426 & .000 \\
\hline
\end{tabular}

Table 1: Pre and posttest for PRTEE, PFGS and PPT of Group A

GROUP B (CONTROL GROUP)

\begin{tabular}{|l|l|l|l|l|l|l|}
\hline \multirow{2}{*}{$\begin{array}{l}\text { OUT COME } \\
\text { MEASURES }\end{array}$} & \multicolumn{2}{l|}{ MEAN } & \multicolumn{2}{l|}{ STANDARD DEVIATION } & \multirow{2}{*}{ Z VALUE } & \multirow{2}{*}{ P VALUE } \\
\cline { 2 - 7 } & PRE TEST & POST TEST & PRE TEST & POST TEST & & \\
\hline PRTEE & 45.60 & 42.63 & 13.333 & 13.631 & -3.450 & .000 \\
\hline PFGS & 14.60 & 16.73 & 4.372 & 4.367 & -3.502 & .000 \\
\hline PPT & 1.95 & 2.16 & .226 & .267 & -3.473 & .000 \\
\hline
\end{tabular}

Table 2: Pre and posttest for PRTEE, PFGS and PPT of Group B 


\section{COMPARISON BETWEEN BOTH GROUPS}

\begin{tabular}{|c|c|c|c|c|c|c|}
\hline $\begin{array}{l}\text { OUTCOME } \\
\text { MEASURES }\end{array}$ & GROUP & $\mathbf{N}$ & MEAN SUM & SUM OF RANKS & $\begin{array}{l}\text { MAN WHITNEY } \\
\text { U TEST }\end{array}$ & P VALUE \\
\hline \multirow[t]{2}{*}{ PRTEE } & $A$ & 15 & 11.87 & 178.00 & \multirow[t]{2}{*}{58} & \multirow[t]{2}{*}{.023} \\
\hline & B & 15 & 19.13 & 287.00 & & \\
\hline \multirow{2}{*}{ PFGS } & $A$ & 15 & 18.57 & 283.00 & \multirow[t]{2}{*}{62} & \multirow[t]{2}{*}{.035} \\
\hline & B & 15 & 12.13 & 182.00 & & \\
\hline \multirow{2}{*}{ PPT } & A & 15 & 22.57 & 338.50 & \multirow[t]{2}{*}{65} & \multirow[t]{2}{*}{.000} \\
\hline & B & 15 & 8.43 & 126.50 & & \\
\hline
\end{tabular}

Table 3: Comparison Of Post Test values of PFGS, PRTEE and PPT Between Group A And Group B

The above pre and posttest mean value tables 1 and 2 shows that both the groups show improvement in pain free grip strength, pressure pain threshold and functional disability. Table 3 shows that Group-A with MWM, MFR and conventional treatment shows better improvement than group-B subjects with conventional treatment.

\section{DISCUSSION}

The purpose of this study was to examine the usefulness of the addition of Mulligan's mobilization with movement and myofascial release technique to recurrent chronic lateral epicondylalgia patients in the age group of 2550 years, in improving painfree grip strength, pressure pain threshold and functional disability.
30 subjects fulfilling the inclusion criteria were included in the study and they were divided into two groups i.e., Group A and Group B with 15 subjects in each group. Each subject was well explained the procedure of the intervention and the possible risks involved. A written informed consent from each subject was obtained. Group A received Mulligan' mobilization with movement, and myofascial release technique in addition to Conventional treatment ${ }^{14}$. Group B received Conventional treatment only. All subjects well tolerated the interventions given and no one was dropped out of the study.

Hand Dynamometer was used for measuring Pain Free Grip Strength, Pressure Algometer for measuring pressure pain threshold and Patient Rated Tennis Elbow Evaluation Questionnaire for measuring functional disabiltiy. The 
affected arm of the subjects was used for the evaluation purpose.

All outcome measures were collected before and after the intervention protocol. Statistical analysis was done using software SPSS16 Version. In both groups, Wilcoxon signed rank test was used to compare pre and posttest values. The post test scores of both groups were analysed using Mann whitney u test. The results showed that after four weeks of mulligan's MWM and MFR technique, there was improvement in pressure pain threshold, pain free grip strength and functional disability in the participants.

CLE is a condition characterized by persistent pain, sensorimotor dysfunction, motor cortex reorganization and muscle imbalance. This results in pain, decreased grip strength and functional disability in recurrent chronic lateral epicondylalgia patients. Therefore recovery in these patients can be assessed using these parameters as outcome measures. Since CLE is a condition with complex aetiological and pathophysiological processes, a multimodal treatment approach which addresses articular, myofascial and nervous system dysfunction will be an effective treatment for recurrent chronic lateral epicondylalgia. Therefore, this study used a multimodal treatment which included Mulligan's MWM, MFR and conventional treatment together for recovery in recurrent chronic lateral epicondylalgia patients.

Hafizur Rahman et $\mathrm{al}^{13}$ shows in his comparitative study between MWM and exercise programme that 4 weeks treatment using Mulligan's MWM and supervised exercise programme are effective in reducing pain and increasing hand grip strength in patients with $L E$, but significant improvement is noted in patients of MWM group compared to exercise programme group. JH Abbott et $\mathrm{al}^{4}$ found that MWM is a useful intervention modality in the rehabilitation of patients with LE. Arun et $\mathrm{al}^{15}$ conducted a study on the effectiveness of MFR therapy along with active stretching and concluded that adding MFR with active stretching helps to reduce pain and improve functional performance in CLE patients. M S Ajimsha et al ${ }^{11}$ conducted a study to investigate whether MFR reduces the pain and functional disability of LE in comparison with a control group receiving sham ultrasound therapy in computer professionals. The PRTEE scale was used to assess pain severity and functional disability. This study provides evidence that MFR is more effective than a control intervention for LE in computer professionals.

The result of the present study supports the above studies. PRTEE was a reliable, reproducible, and sensitive instrument for assessment of chronic lateral epiondylalgia. The objective of using the patient-rated tennis elbow evaluation questionnaire (PRTEE) in this study was to provide an uncomplicated, standardized, quantitative description of pain and functional disability in recurrent chronic lateral epicondylalgia patients before and after the interventions in Group A and Group B. Based on the statistical analysis in experimental group (Group A), the pretest mean value with standard deviation of PRTEE was $42.63 \pm 13.102$ with minimum value of 22 and maximum value of 65 and the posttest mean value with standard deviation of PRTEE was $31.03 \pm 12.709$ with minimum value of 13 and maximum value of 54 , the mean difference was $11.60, z$ value was -3.415 and $p$ value was 0.000 . The result of the study shows that there is statistically significant difference between pretest and posttest values of PRTEE in experimental group. Based on the statistical analysis in Control 
group (Group B), The pretest mean value with standard deviation of PRTEE was $45.60 \pm 13.333$ with minimum value of 29 and maximum value of 68 and the posttest mean value with standard deviation of PRTEE was $42.63 \pm 13.631$ with minimum value of 25 and maximum value of 66 , mean difference was 2.97 , $z$ value was 3.450 and $p$ value was 0.000 . The result of the study shows that there is statistically significant difference between pretest and posttest values of PRTEE in Control group (Group B). While comparing Group A and Group B, the mean rank for group $A$ was 11.87 and for group $B$ was 19.13. The sum of rank for group $A$ was 178 and for group B was 287, Mann Whitney $U$ value was 58 , the $P$ value was 0.023 . The result of the study shows that there is a statistically significant difference between the posttest mean rank of PRTEE in Group A and Group B. The posttest mean rank of PRTEE shows that Experimental Group (Group A) shows significant improvement in functional disability than Control Group (Group B). Newcomer et al evaluated the sensitivity, reliability and concurrent validity of the PRTEE on 22 subjects with chronic tennis elbow of $>3$ months on 3 consecutive days who were partaking in a concurrent trial investigating the effect of exercise on lateral epicondylitis. The outcome trial compared the PRTEE, VAS, PFG, Disabilities of the arm, shoulder and hand questionnaire and Medical Outcomes Study 36-item Short Form Health Survey and concluded that it will assist the clinician in understanding the impact of tennis elbow on a patient.

Amrish O. Chourasia et al ${ }^{16}$ says that ability to rapidly generate grip force is affected in LE. The grip strength is affected due to voluntary decline of effort to avoid pain and due to wasting of affecting muscles seen in long standing conditions.
Marc-Andre Blanchette et $\mathrm{al}^{17}$ found that Pain Free Grip strength is the most indicated strength measurement to monitor recovery of patients with lateral epicondylalgia. The objective of using the pain free grip strength in this study before and after the interventions in Group A and Group B was it is one of the most valid outcome measures of change over time in recurrent chronic lateral epicondylalgia patients. Based on the statistical analysis in experimental group (Group A), The pretest mean value with standard deviation of PFGS was $12.80 \pm 3.688$ with minimum value of 7 and maximum value of 19 and the posttest mean value with standard deviation of PFGS was $20.47 \pm 3.907$ with minimum value of 15 and maximum value of 27 , the mean difference was $7.67, z$ value was -3.423 and $p$ value was 0.000 .

The result of the study shows that there is statistically significant difference between pretest and posttest values of PFGS in experimental group. Based on the statistical analysis in Control group (Group B), The pretest mean value with standard deviation of PFGS was $14.60 \pm 4.372$ with minimum value of 8 and maximum value of 22 and the posttest mean value with standard deviation of PFGS was $16.73 \pm 4.367$ with minimum value of 10 and maximum value of 24 , mean difference was $2.13, z$ value was -3.502 and $p$ value was 0.000 . The result of the study shows that there is statistically significant difference between pretest and posttest values of PFGS in Control group (Group B). While comparing Group $A$ and Group $B$, the mean rank for group $A$ was 18.87 and for group $B$ was 12.13. The sum of rank for group A was 283 and for group B was 182 , Man Whitney $U$ value was 62 , the $P$ value was 0.035 . The result of the study shows that there is a statistically significant difference between the posttest mean rank of PFGS in 
Group A and Group B. The posttest mean rank of PFGS shows that Experimental Group (Group A) shows significant improvement in pain free grip strength than Control Group (Group B). The use of PFG as an objective quantitative outcome measure has been endorsed by a number of studies.

Smidt et $\mathrm{al}^{18}$ recommended using PFGS due to it not only being reliable and relatively easy to execute but that it has been associated with other measures of functional disability. Stratford et al. evaluated a number of outcome measures on a representative sample of 40 patients with tennis elbow. They found that PFG and pain free function questionnaire (PFFQ) were the most valid outcome measures of change over time in tennis elbow patients. High reliability coefficients for PFGS (0.87) and PFFQ (0.93) had previously been reported.

Ann $M$ Kinser et $\mathrm{al}^{19}$ states that algometers are devices that can be used to identify the pressure and / force eliciting a pressure pain threshold. He concluded that painful regions have decreased PPT and algometers are the useful way to quantify pain and possibly track recovery / healing in LE patients. Therefore this study used pressure pain threshold as one outcome and measured using pressure algometer before and after the interventions in Group A and Group B. Based on the statistical analysis in experimental group (Group A), The pretest mean value with standard deviation of PPT was 2.20 \pm 0.469 with minimum value of 2 and maximum value of 3 and the posttest mean value with standard deviation of PPT was $3.09 \pm 0.425$ with minimum value of 2 and maximum value of 4 , the mean difference was $0.89, z$ value was -3.426 and $p$ value was 0.000 . The result of the study shows that there is statistically significant difference between pretest and posttest values of PPT in experimental group. Based on the statistical analysis in Control group (Group B), The pretest mean value with standard deviation of PPT was $1.95 \pm 0.226$ with minimum value of 2 and maximum value of 2 and the posttest mean value with standard deviation of PPT was 2.16 \pm 0.267 with minimum value of 2 and maximum value of 3 , mean difference was $0.21, z$ value was -3.473 and $p$ value was 0.000 . The result of the study shows that there is statistically significant difference between pretest and posttest values of PPT in Control group (Group B). While comparing Group A and Group B, the mean rank for group $A$ was 22.57 and for group $B$ was 8.43.The sum of rank for group $A$ was 338.50 and for group B was 126.50 , Mann Whitney $U$ value was 6.5 , the $P$ value was 0.000 . The result of the study shows that there is a statistically significant difference between the posttest mean rank of PPT in Group A and Group B. The posttest mean rank of PPT shows that Experimental Group (Group A) shows significant improvement in Pressure pain threshold than Control Group (Group B).

Beatriz Ruiz-Ruiz ${ }^{20}$ states that the most sensitive localizations for PPT assessment were found in locations corresponding to the muscle belly of the ECRB in CLE patients.

The interventions used in this study are Muliigan's MWM, MFR and conventional treatment. The proposed mechanism lying behind Mulligan's MWM is of two concepts. One is positional fault and other is neurophysiological concept. A positional fault means condition in which the joint surface is not in a natural and congruent position, and it is not easily palpated nor readily detected by radiological examination. Such positional faults result in damage and strains. Therefore, the 
correction of positional fault through MWM encourages normal joint motion and joint fluid flow inducing recovery. MWM is considered to suppress excitation of the muscular origins of lateral epicondyle, and correct the positional fault of the humerus resulting from protective muscle spasms, thereby inducing normal arthrokinematic movement.

Mulligan's MWM is a useful technique for eliminating the pain of a previously painful active movement, in patients with chronic lateral epicondylalgia. It resulted in a significant increase in pain-free grip strength in chronic lateral epicondylalgia subjects. Mulligan's MWM performed for CLE takes place in two stages. First, therapist applies lateral gliding force to the patient's elbow, which has the effect of stretching the origin muscles of the lateral epicondyle .Second, the therapist applies passive lateral gliding force to the elbow while the patient actively makes a fist, and the patient's motion stretches the origin muscles of the lateral epicondyle. Therefore, muscle strengthening using these two treatment elements is considered to stimulate the Golgi tendon organs [GTO] in the tendons of these muscles.GTOs are positioned in series against contractile elements, and therefore they are stimulated both when the muscles are stretched and when they are contracted .GTO signals are transmitted to the spinal cord through the $\mathrm{Ib}$ afferent fiber, and suppress motor neurons through the synapses by means of inhibitory interneurons. Therefore major treatment focus of MWM is the correction of positional fault. This provides an alternative to the theories offered by Mulligan and Exelby, which place a positional fault or block of the joint as the source of the dysfunction in LE.
Myofascial release technique is effective in reducing pain and disability and in improving grip strength in chronic lateral epicondylalgia. MFR is more effective when compared with active release technique in CLE patients. MFR can alleviate fascial restrictions in CLE patients. With all the above findings it has been proved that the results of the present study have shown similar results with other studies which have been done using Mulligan's MWM and MFR in treatment of recurrent chronic lateral epicondylalgia.

\section{Limitations of the study}

- The maintenance and enhancement of long term treatment effects were not studied.

- Hand dynamometer used in this study was not very precise as it is manual hand dynamometer.

- Pressure Algometer used in this study was manual algometer.

- This study didn't include the effect of psychological factors on recovery in recurrent chronic lateral epicondyllgia subjects.

- Small sample size

\section{Recommendations}

- Larger study involving an increased number of participants should be included.

- Further studies to determine long term effect of these techniques can be appreciated.

- Use of an electronic digital dynamometer is suggested to be used to measure grip strength as it may be more accurate.

- Use of an electronic pressure algometer is suggested to be used to measure grip strength, as it may be more accurate. 
- Future studies including effect of psychological factors in recovery in CLE patients are warranted.

- RCT can be done; the study should be repeated as double or single blinded study. This would eliminate any possible bias and allow for all patients to be treated in exactly the same manner, eliminating any possible placebo effects.

\section{CONCLUSION}

Based on the statistical analysis, the result of the present study shows that there is statistically significant difference in pain, grip strength and functional disability between pretest and post-test in both experimental and control group. Experimental group shows greater improvement in pain while measuring pressure pain threshold, grip strength while measuring using hand dynamometer and functional disability while measuring patient rated tennis elbow evaluation questionnaire than control group in subjects with recurrent chronic lateral epicondylalgia.

Thus the study concludes that Mulligan's mobilization with movement technique with myofascial release technique is very effective for improving pressure pain threshold, grip strength and functional disability in recurrent chronic lateral epicondylalgia patients.

\section{REFERENCES}

1. Ratan Khuman, Parth Trivedi, Surbala Devi, D. Sathyavani, Gopal Nambi, Kimi Shah. (2013). Myofascial Release Technique in Chronic Lateral Epicondylitis: A Randomized Controlled Study.
International Journal of Health Sciences \& Research 3(7):45-52.

2. Stasniopoulos D, Johnson MI (2005).Effectiveness of Extracorporeal Shock Wave therapy for Tennis elbow. Br J of Sports Med 39: 132-136.

3. Brukner, Bahr, Blair,Cook, Crossley, McConnell et al(2012). Clinical sports medicine-4rd ed; McGraw-Hill; 393-400.

4. Abbott JH, Patla CE, Jensen RH (2001). The initial effects of an elbow mobilisation with movement technique on grip strength in subjects with lateral epicondylalgia. Manual Therapy 6(3):163169.

5. Assendelft WJ, Hay EM, Adshead R, Bouter LM (1996). Corticosteroid injections for lateral epicondylitis: a systematic overview. Br J Gen Pract; 46: 209-16.

6. Shiri R, Viikari-Juntura E, Varonen $H$, Heliovaara M (2006). Prevalence and determinants of lateral and medial epicondylitis: a population study. Am J Epidemiol; 164: 1065-74.

7. Smidt N, Lewis M, Hay EM, Buter LM, Craft $P(2006)$.Lateral epicondylitis in general practice- Corse and Prognotic indicators of outcome. Journal of Rheumatology; 33(10):2053-59.

8. Hong QN, Durand MJ, Loisel P (2004). Treatment of lateral epicondylitis: where is the evidence? .Joint Bone Spine: 71:36973.

9. Mulligan BR (1993). Mobilisation with movement. J Man Manip Ther;1(4):154e6.

10. Atanda A Jr, Shah SA, O'Brien K (2011).Osteochondrosis: common causes of pain in growing bones. Am Fam Physician 83(3):285-29.

11. Ajimsha $M$, Chithra $S$, Thulasyammal $R$ (2012). Effectiveness of myofascial release in the management of lateral epicondylitis 
in computer professionals. Archives of physical medicine and rehabilitation; 93 (4): 604-609.

12. Nirschl RP, Ashman ES (2003). Elbow tendinopathy- tennis elbow, Clin Sports Med; 22:813-836.

13. Hafizur Rahman, Pilladi A Chaturvedi, Patchava Apparao, Pilladi R Srithulasi(2016). Effectiveness of mulligan mobilisation with movement compared to supervised exercise program in subjects with lateral epicondylitis; International Journal of Physiotherapy and Research, Int J Physiother Res, Vol 4(2): 1394-1400.

14. Parth Trivedi , D. Sathiyavani , Gopal Nambi , Ratan Khuman, Kimi Shah ,Preeti Bhatt (2014) Comparison Of Active Release Technique And Myofascial Release Technique On Pain, Grip Strength \& Functional Performance In Patients With Chronic Lateral Epicondylitis, International Journal Of Physiotherapy And Research, Int J Physiother Res, Vol 2(3): 488-94.

15. Arun Balasubramaniam , Mohanraj Kandhasamy(2016).Effect of Myofascial Release Therapy and Active Stretching on Pain and Grip Strength in Lateral Epicondylitis, Journal of Riphah College of Rehabilitation Sciences, JRCRS; 4(1): 3-6.

16. Amrish O. Chourasia, Kevin A. Buhr, David P. Rabago, Richard Kijowski, Curtis B. Irwin, Mary E. Sesto( 2012), Effect of Lateral Epicondylosis on Grip Force Development J Hand Ther; 25(1): 27-37.
17. Marc-André Blanchette, Martin C. Normand (2011). Impairment assessment of lateral epicondylitis through electromyography and dynamometry, J Can Chiropr Assoc ; 55(2).

18. Smidt N, Assendelft WJ, van der Windt DA, Hay EM,Buchbinder R,Bouter LM(2002).Corticosteroid injections for lateral epicondylitis;a systematic review. Pain; 96; 23-40.

19. Ann M Kinser, William A Sands, Michael Henry Stone (2009). Reliability and Validity of a Pressure Algometer,Journal of Strength and conditioning research, January, vol 23(1).

20. Beatriz Ruiz-Ruiz, Cesar Fernandez-de-lasPenas, Ricardo Ortega-Santiago, Lars Arendt-Nielsen, Pascal Madeleine (2011). Topographical Pressure and Thermal Pain Sensitivity Mapping in Patients with Unilateral Lateral Epicondylalgia. The Journal of Pain, Vol 12, No 10 (October): pp 1040-1048.

21. Namrata Patel (2013).Effectiveness of mobilization with movement of elbow compared with manipulation of wrist in patients of lateral epicondylitis. Int J Physiother Res, Vol1(4):177-82.

22. MulliganB.Manual.Therapy: NAGS, NAGS, MWMS etc, (2006), $5^{\text {th }}$ ed. Wellington NZ: Plane view services.

\section{Citation:}

Anusha M S, Andrews Milton. Effectiveness of mobilization with movement and myofascial release technique in improving pain free grip strength, pressure pain threshold and functional disability in subjects with recurrent chronic lateral epicondylalgia , IJMAES, 2017; 3 (3), 326-339. 